REVIEW ARTICLE

N. Kumar

\section{Neuroimaging in Superficial Siderosis: An In-Depth Look}

SUMMARY: Despite extensive imaging, a source of bleeding is often not evident during the evaluation of patients with superficial siderosis (SS) of the central nervous system. An intraspinal fluid-filled collection of variable dimensions is frequently seen on spine MR imaging in patients with idiopathic SS. A similar finding has also been reported in patients with craniospinal hypotension. This review discusses the role of multitechnique imaging in the work-up of patients with SS and focuses on recent developments.
S uperficial siderosis (SS) of the central nervous system (CNS) results from hemosiderin deposition in the subpial layers of the brain and spinal cord. ${ }^{1-4}$ The hemosiderin deposition is a consequence of recurrent and persistent bleeding into the subarachnoid space. The ability of the brain to biosynthesize ferritin in response to prolonged contact with hemoglobin iron is important in the pathogenesis of SS. ${ }^{4-7}$ Accelerated ferritin synthesis in the Bergmann glia of the cerebellum may account for preferential cerebellar involvement. $^{5}$

The classic clinical presentation of SS includes adult-onset slowly progressive gait (less commonly appendicular) ataxia with cerebellar dysarthria and sensorineural hearing impairment. ${ }^{1-3}$ The bleeding source may be not be detected despite extensive neuroimaging. Commonly performed investigations during the work-up of SS include MR imaging of the brain and spine, CT myelography, MR angiography, and cerebrospinal angiography. A prior history of trauma (at times trivial) or intradural surgery (commonly involving the posterior fossa) may be present. ${ }^{2,8-18}$ Often decades elapse between the presumed inciting event and the development of symptoms attributable to SS.

Brain MR imaging is the investigation of choice for the diagnosis of SS. ${ }^{2,3,8,19-31}$ Before the advent of MR imaging, approximately 40 cases had been reported in the world literature. $^{32}$ The diagnosis of SS had largely been postmortem or made during surgical exploration. ${ }^{32-34}$ With widespread use of MR imaging, presymptomatic cases are being diagnosed, and it is difficult to be certain about the true incidence of the disorder. $^{25}$ In vivo MR imaging and postmortem correlation have shown that the hemosiderin deposition around the brain, brain stem, and spinal cord is the cause of the characteristic hypointensity seen on T2-weighted MR imaging. ${ }^{8}$

In recent years, an extra-arachnoid longitudinally extensive intraspinal fluid-filled collection has been frequently noted in patients with SS..$^{2,3,28-31,35,36}$ A CT myelogram, dynamic CT myelogram, or digital substraction myelogram can identify a dural defect that connects the intrathecal space with the fluid-filled collection. ${ }^{29,35,36}$ The precise mechanism of bleeding in these cases is unknown. Limited experience sug-

From the Department of Neurology, Mayo Clinic, Rochester, Minnesota.

Please address correspondence to Neeraj Kumar, MD, Department of Neurology, Mayo Clinic, 200 First St SW, Rochester, MN 55905; e-mail: kumar.neeraj@mayo.edu

Indicates open access to non-subscribers at www.ajnr.org

DOI 10.3174/ajnr.A1628 gests that repair of the defect may be associated with clinical stability or improvement, resolution of the intraspinal fluidfilled collection, and resolution of CSF evidence of subarachnoid bleeding. ${ }^{2,28,29,31,36}$ Some reports have also noted an association between SS and CSF hypovolemia and low-pressure headache. ${ }^{30,31,35}$ CSF hypovolemia (craniospinal hypotension) is a disorder that is frequently associated with dural defects, occasionally with intraspinal fluid collection of variable longitudinal extent and rarely with red blood cells (RBCs) or xanthochromia in the CSF. ${ }^{37-40}$

The surgical treatment of SS depends on early identification of the bleeding source. Surgical excision of the offending lesion (neoplasm or vascular malformation or pseudomeningoceles) and repair of dural defects are logical therapeutic strategies.

This review discusses the role of neuroimaging in the diagnosis and management of SS and focuses on recent developments in the understanding of this disorder. All the images of patients with SS shown in this review are from patients seen at the Mayo Clinic, except for Fig $8 E$.

\section{MR Imaging and the Diagnosis of SS}

In the early stages of SS, the findings are subtle and a high index of suspicion is necessary to establish a diagnosis. The T2 hypointensity follows the contours of the brain and spinal cord and may be easily missed (Figs $1 A-I ; 2,3 A,-B ; 4 B,-E$ ). Imaging of the entire neuraxis is indicated to localize a potential bleeding source. Gradient-echo T2-weighted images have a higher sensitivity for hemosiderin deposition (Fig $2 A,-B$ ). The magnetic susceptibility effects of blood-degradation products such as ferritin and hemosiderin are also more pronounced at higher field strengths.

T2-weighted MR imaging shows a rim of hypointensity around the cerebellum (Fig $1 A,-B$ ) and brain stem (Fig $1 C-E$ ). The marginal $\mathrm{T} 2$ hypointensity may also involve the Sylvian fissure (Fig $1 F$ ), interhemispheric fissure (Fig $1 G$ ), and cortical sulci (Fig $1 H$ ). The superior vermis, quadrigeminal plate, and basal cerebral surface are preferentially affected by the marginal T2 hypointensity. Hemosiderin deposition may also be seen along cranial nerves II, V, VII, and VIII (Fig 1I). The peripheral nervous system is not involved in SS. There is a sharp cutoff in the spinal roots and cranial nerves at the junction between the peripheral Schwann cell segment and the central glial segment. ${ }^{41}$ Cerebellar atrophy is often present, and the superior vermis and anterior cerebellar hemispheres may be preferentially involved by the atrophy (Fig $1 J$ ). The gliosis and neuronal loss associated with hemosiderin deposi- 

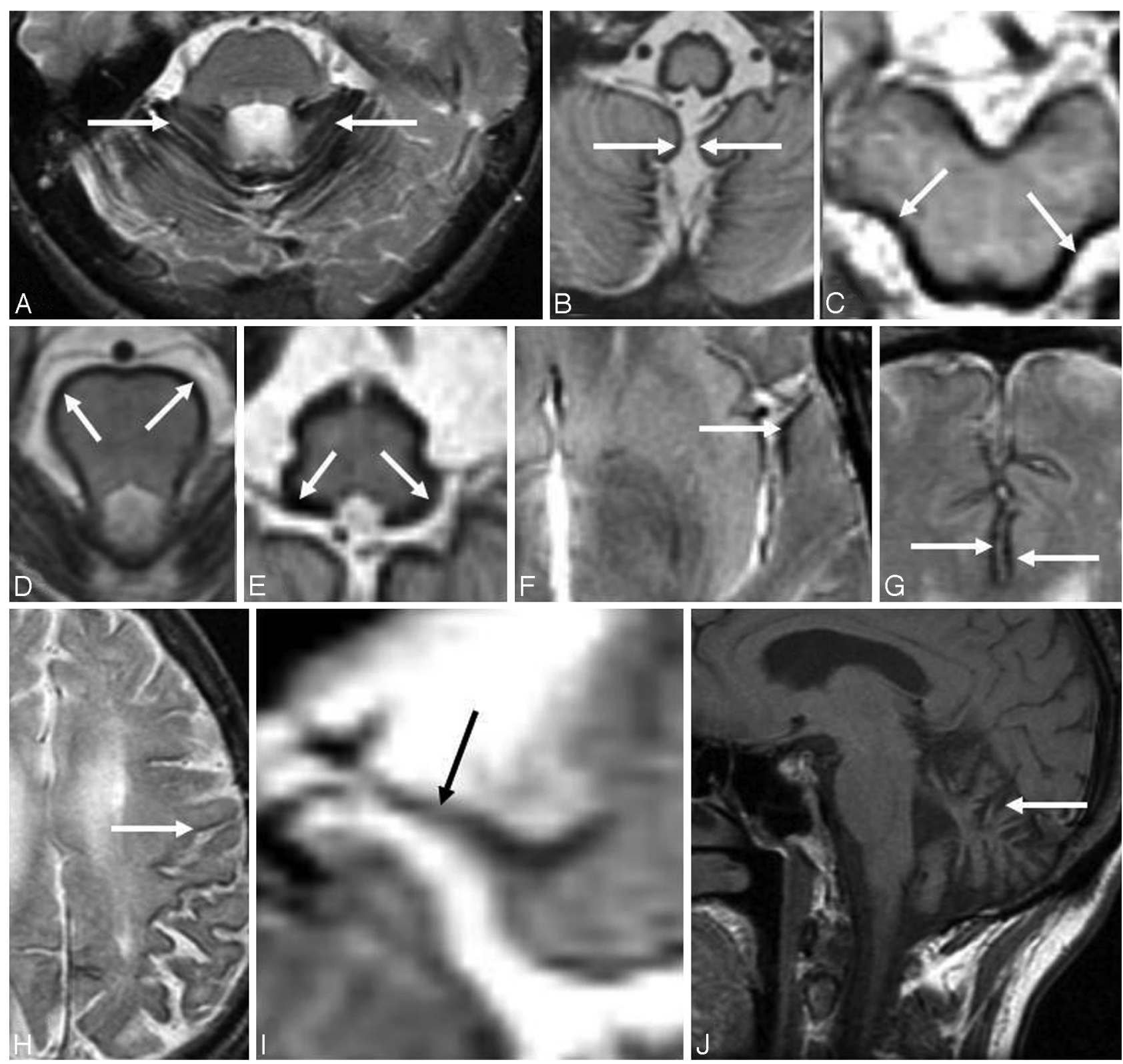

Fig 1. $A-I$, Axial T2-weighted brain MR images from patients with SS show hemosiderin deposition along the cerebellar folia $(A)$, vermis $(B)$ and around the midbrain $(C)$, pons $(D)$, medulla $(E)$, Sylvian fissure $(F)$, interhemispheric fissure $(G)$, cerebral convexity $(H)$, and the course of the eighth cranial nerve $(\Lambda)$. J, Sagittal T1-weighted brain MR image from a patient with SS shows severe cerebellar atrophy.
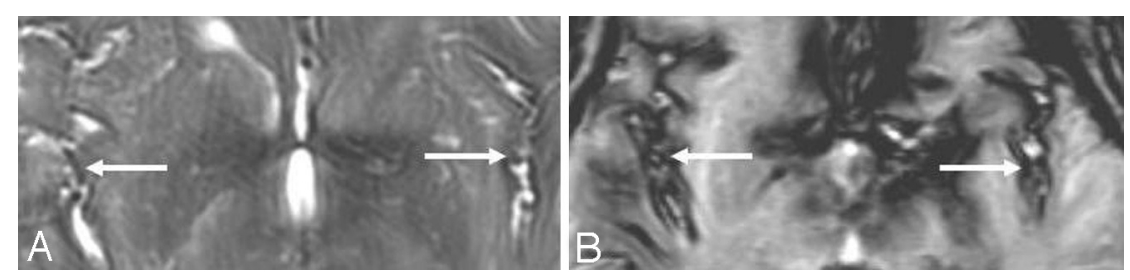

Fig 2. T2-weighted $(A)$ and gradient-echo $\left(\mathrm{T}^{*}\right)(B) \mathrm{MP}$ images from a patient with SS demonstrate superiority of the gradient-echo technique in detecting the characteristic T2 hypointensity, shown here along the Sylvian and interhemispheric fissures. Both images are from the same patient and were obtained at the same point in time. Reprinted with permission from Kumar N. Superficial siderosis: associations and therapeutic implications. Arch Neurol 2007;64:491-96 (Copyright 2007, American Medical Association). tion may result in increased signal intensity from the adjacent cerebellar tissue. ${ }^{22,26}$ Alternatively, it may be only an apparent increase due to the overlying hypointensity. Rarely, a hyperintense rim may be seen on T2-weighted imaging. ${ }^{26}$ This may be due to methemoglobin and represents a recent episode of subarachnoid hemorrhage.

Hemosiderin deposition is commonly seen around the spinal cord (Fig $3 A,-B)$. Spinal cord atrophy is common (Fig $3 A$ ).
There may be peripheralization (Fig 3C) or clumping (Fig 3D; also seen on a CT myelogram in Fig $3 E$ ) of the nerve roots due to chronic subarachnoid bleeding-related arachnoiditis. Blood accumulation with reactive changes in the cul-de-sac may give the mistaken impression of a tumor (Fig $3 F) .{ }^{3}$ The leptomeninges in SS are fibrotic, thickened, and have hemosiderin-laden macrophages. ${ }^{42}$ Very rarely, increased signal intensity due to presumed calcification at sites of hemosiderin 

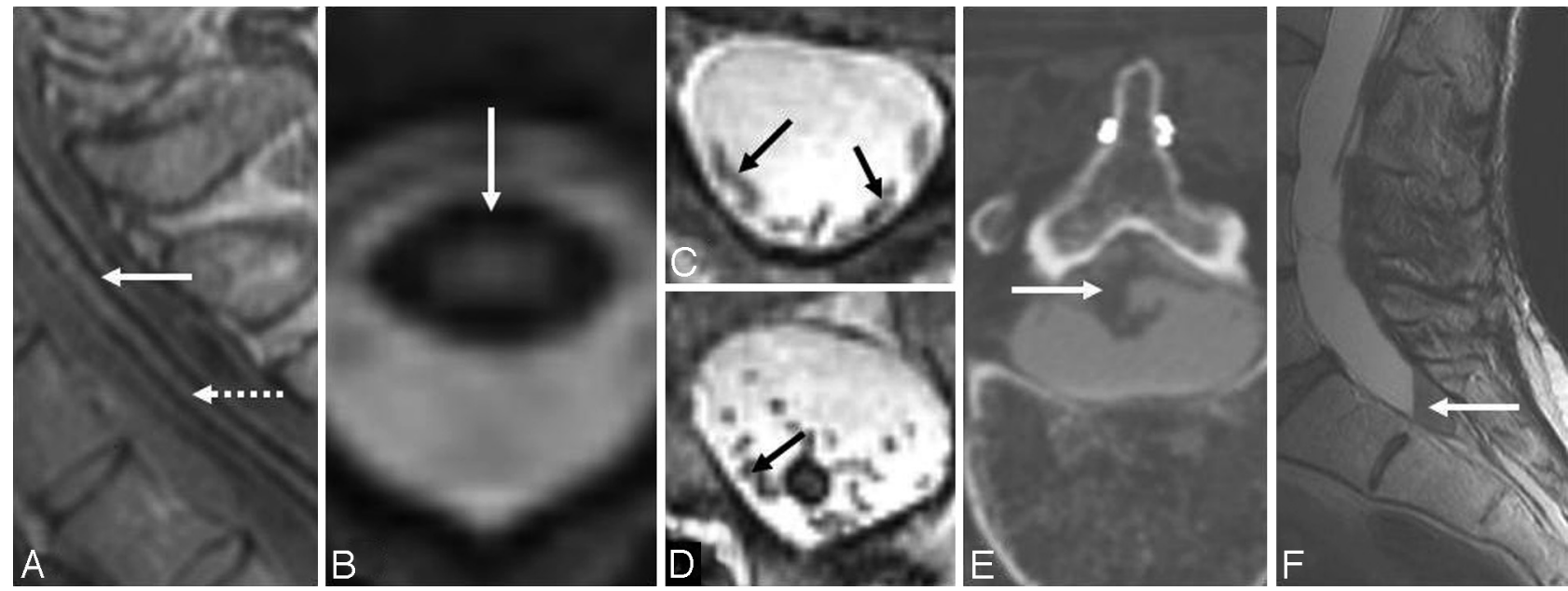

Fig 3. $A$ and $B$, Sagittal $(A)$ and axial $(B)$ T2-weighted spinal cord MR images show hemosiderin deposition along $(A)$ and around $(B)$ the cord surface. Note associated severe cord atrophy (A) (dotted arrow). $C$ and $D$, Axial T2-weighted MR images at the level of the cauda equina from patients with SS show peripheralization (C) and clumping $(D)$ of the nerve roots due to arachnoiditis. $E$, An axial cut at the lumbar levels on a CT myelogram from a patient with SS shows clumping of nerve roots of the cauda equina due to arachnoiditis. $F$, T2-weighted sagittal MR image of the lumbosacral area from a patient with SS shows a lesion that was suspected of being a possible source of the chronic bleeding. A biopsy was performed, and blood products and fibrous tissue were detected. $C$ and E reprinted with permission from Kumar N. Superficial siderosis: associations and therapeutic implications. Arch Neurol 2007;64:491-96 (Copyright 2007, American Medical Association).
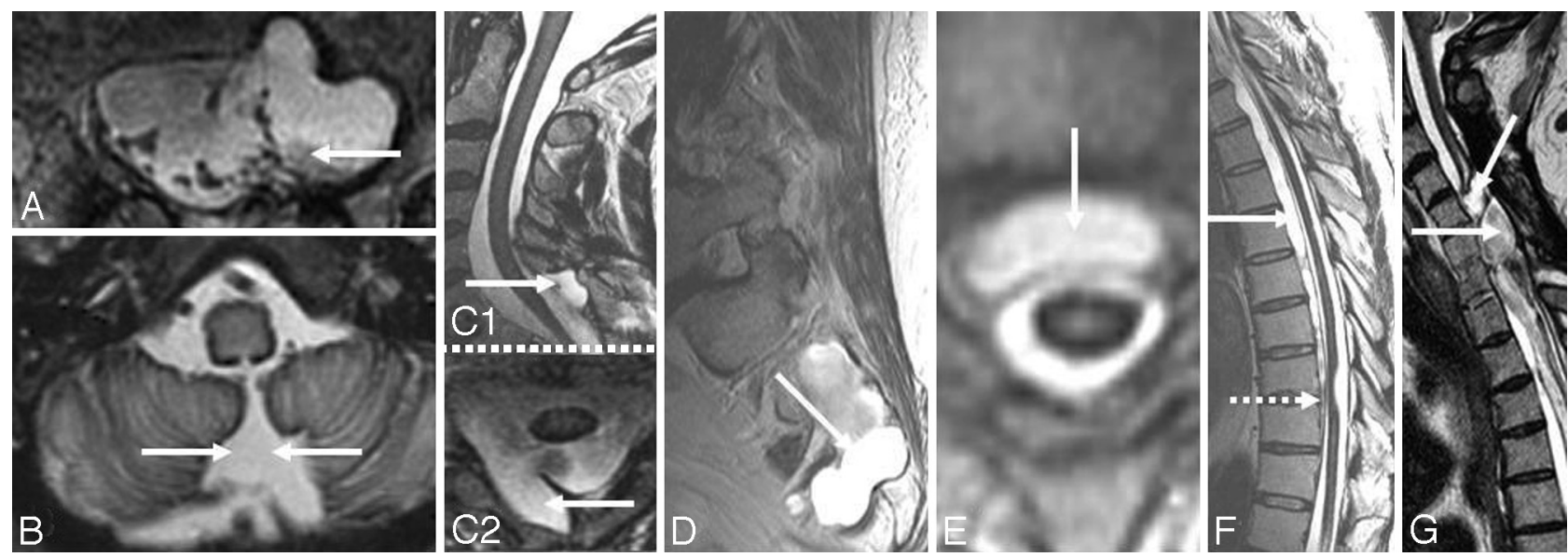

Fig 4. A, Axial T2-weighted spine MR image from a patient with SS shows a left T12 pseudomeningocele. $B$, Axial T2-weighted brain MR image from a patient with SS shows a posterior fossa fluid-filled collection. C1 and C2, Sagittal (C1) and axial (C2) T2-weighted cervical MR images from a patient with SS show a right C7 pseudomeningocele (same patient as the one shown in Fig 10B). D, Sagittal T2-weighted spine MR image from a patient with SS shows an intrasacral meningocele. E, Axial T2-weighted thoracic cord MR image shows a fluid-filled collection anterior to the spinal cord. F, Sagittal T2-weighted MR image shows a longitudinally extensive fluid collection ventral to the cord. The dotted arrow shows tethering of the cord at T9. G, Sagittal T2-weighted spine MR image from a patient with SS shows multiple intraspinal fluid-filled loculations. C1 and C2 reprinted with permission from Kumar N, Cohen-Gadol AA, Wright RA, et al. Superficial siderosis. Neurology 2006;66:1144-52 (Copyright 2006, Wolters Kluwer Health). F reprinted with permission from Wilden JA, Kumar N, Murali HR, et al. Unusual neuroimaging in superficial siderosis. Neurology 2005;65:489 (Copyright 2005, Wolters Kluwer Health).

deposition may be seen on the cord surface on a noncontrast T1-weighted MR imaging (shown on a CT scan in Fig 5C).

An indirect clue to the etiology of SS may be evident on MR imaging. A fluid-filled intraspinal cavity of variable dimension is often seen (Fig $4 A,-C 1$ and $C 2, D-G$; Fig $6 A 1$ and $A 2, B 1$ and $B 2$; Fig $7 A 1$ and $A 2$; also seen on the CT myelogram in Fig $7 B 1$ and $B 2$ and Fig 10A).,2,28-31,35,36 Rarely the fluid-filled cavity may be intracranial (Fig $4 B$ ). ${ }^{43}$ These localized fluidfilled cavities have been variably referred to as meningoceles, pseudomeningoceles, diverticula, pseudoencephalocele, epidural cyst, or simply as "fluid collections." Nerve root avulsions, often in association with brachial plexus injury and pseudomeningoceles, are a commonly reported association. ${ }^{2,10,11,14,17,27,44}$ An interruption in the circumferential hypointensity on axial T2-weighted MR imaging may indicate root avulsion and is possibly due to absence of the pia mater at the avulsion site (Fig $8 A$; also seen on a CT myelogram in Fig $10 C) .{ }^{27}$ Evidence of bony injury (Fig $8 C$; also seen on the CT scan in Fig 10B), myelomalacia (Fig $8 D$ ), or cord tethering (Fig $4 F)$ may also be present. The other identified sources of bleeding in patients with SS have typically included neoplasms and vascular malformations. Cerebral amyloid angiopathy may also be associated with evidence of hemosiderin deposition similar to that seen in SS (Fig 8E). ${ }^{45}$

In a 1995 survey of the reported SS cases in the world literature, an underlying cause was identified in 34 of 63 cases. ${ }^{1}$ A dural pathology was found in $47 \%$. This included CSF cavity lesions (such as meningocele, pseudomeningocele, pseudoencephalocele, a cavity remaining after a hemispherectomy, and chronic suboccipital hematoma) or root pathology (such as root avulsion or epidural cyst). Tumors such as ependymoma, meningioma, oligodendroglioma, pineocytoma, and paragan- 

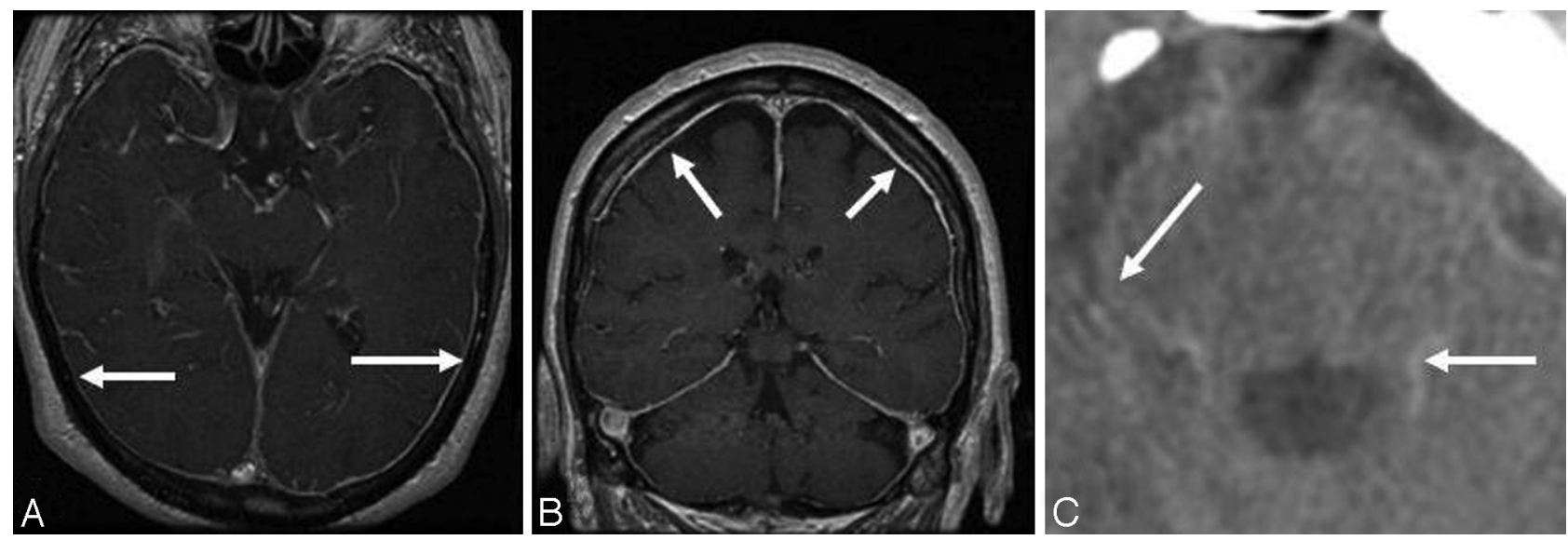

Fig 5. $A$ and $B$, Axial $(A)$ and coronal postcontrast $(B)$ T1-weighted MR images from a patient with SS show diffuse pachymeningeal enhancement similar to that reported in patients with craniospinal hypotension. (same patient as shown in $11 A 1$ and $A 2$ ). $C$, Axial CT scan from a patient with SS shows increased attenuation around the pons due to presumed calcification of the hemosiderin deposition. $A$ and $B$ reprinted with permission from Kumar N, McKeon A, Rabinstein AA, et al. Superficial siderosis and CSF hypovolemia: the defect (dural) in the link Neurology 2007;69:925-26 (Copyright 2007, Wolters Kluwer Health).

glioma were seen in 35\%, and vascular abnormalities such as arteriovenous malformation or aneurysm, in $18 \%$. In a recently reported single-institution series of 30 patients, a fluidfilled collection was seen on MR imaging in $14 .^{2}$ In 4 of these, the collection was fairly localized and suggested the possibility of a meningocele or pseudomeningocele. Longitudinally extensive fluid-filled collections were seen in the others and are a recently identified association of SS. . $^{2,28-31,36}$

Clinical or electrophysiologic evidence of lower motor neuron involvement is rare in SS but has been described and may be due to arachnoiditis or radiculopathy; the latter may result from stretching of nerve roots by intraspinal cysts. ${ }^{17,46-49}$

Some patients with SS and intraspinal fluid-filled collections may have diffuse pachymeningeal enhancement on postcontrast MR imaging (Fig $5 A,-B) .{ }^{30,31}$ This is identical to the pachymeningeal enhancement seen in patients with craniospinal hypotension, a condition also associated with intraspinal fluid-filled collections (Fig 9A-C1). ${ }^{38}$ The cause of intracranial pachymeningeal enhancement in craniospinal hypotension is believed to be vascular dilation within the dura mater rather than meningeal inflammation. ${ }^{50-52}$ A similar mechanism is likely operative in those patients with SS who have a dural defect and an intraspinal fluid-filled collection. Craniospinal hypotension may be associated with RBCs in the CSF. RBCs in the CSF are a defining feature of SS. The presence of RBCs in the CSF in both conditions may be due to a similar mechanism. The longitudinal fluid-filled intraspinal collection can resolve with repair of the dural defect (Fig 6A1 and $A 2, B 1$ and B2).

\section{CT, Postmyelogram CT, and Dynamic CT Myelogram}

Although the head or spine CT findings are generally unremarkable, cerebellar atrophy or a clue to the potential etiology of the SS may be evident. ${ }^{53,54}$ Postcontrast CT in SS has rarely been associated with widespread meningeal enhancement. ${ }^{55}$ Evidence of bony injury due to prior trauma, if present, may be better seen on CT than on MR imaging (Fig 10B). Rarely, a hyperattenuated rim due to calcification at sites of hemosiderin deposition around the brain stem may be seen on CT (Fig $5 C)^{22}$

Dural diverticula, pseudomeningoceles, root avulsions, and associated dural defects are best characterized on myelography, CT myelography, or dynamic CT myelography (Fig $10 C,-D, E 1$ and $E 2, F 1$ and $F 2$ ). CT myelography may also show clumping of nerve roots as evidence of SS-related arachnoiditis (Fig 3E). Recent reports have also noted calcified disk protrusions adjacent to dural tears in patients with SS (Fig 7B1 and B2, Fig 10E2; also seen on MR imaging in Fig 7A1 and A2) or low-pressure headache (Fig 10F2)..$^{31,35,36} \mathrm{CT}$ myelography may show these abnormalities even when the MR imaging findings are unrevealing. ${ }^{2}$

A CT myelogram can also be used to identify the site of leak in those patients with SS who have a longitudinally extensive fluid-filled intraspinal collection and a suspected dural tear. A limitation of the study is its poor temporal resolution. During the time between intrathecal contrast injection under fluoroscopy and acquisition of the CT images, there occurs equilibration of contrast between the intradural and extradural CSF collections. This is particularly true with large defects or multiple defects or high-flow leaks. Dynamic CT myelography by using multidetector CT and a workstation capable of producing multiplanar reconstructions provides sufficient temporal and spatial resolution and can overcome the limitation seen with routine myelography or routine myelography with delayed CT imaging directed at detecting slow-flow leaks. The technique of dynamic CT myelography was introduced for detection of leaks in patients with spontaneous intracranial hypotension. ${ }^{56}$ More recently, it has been used to identify dural defects connecting the intrathecal space with the intraspinal fluid-filled collection seen in patients with SS (Fig 10D, E1 and E2) or low-pressure headache (Fig 10F1). ${ }^{29}$

\section{Angiography}

Cerebral and spinal angiography are commonly undertaken investigations directed at finding an occult source of bleeding. These are generally unrewarding. This may be due to the slow and intermittent nature of the bleeding. Further vascular malformations as a cause of chronic bleeding in SS are rare. The detection of a vascular malformation during the work-up of a patient with SS does not necessarily mean that the aneurysm or arteriovenous anomaly is the source of bleeding. This is particularly so for incidentally detected venous angiomas or 

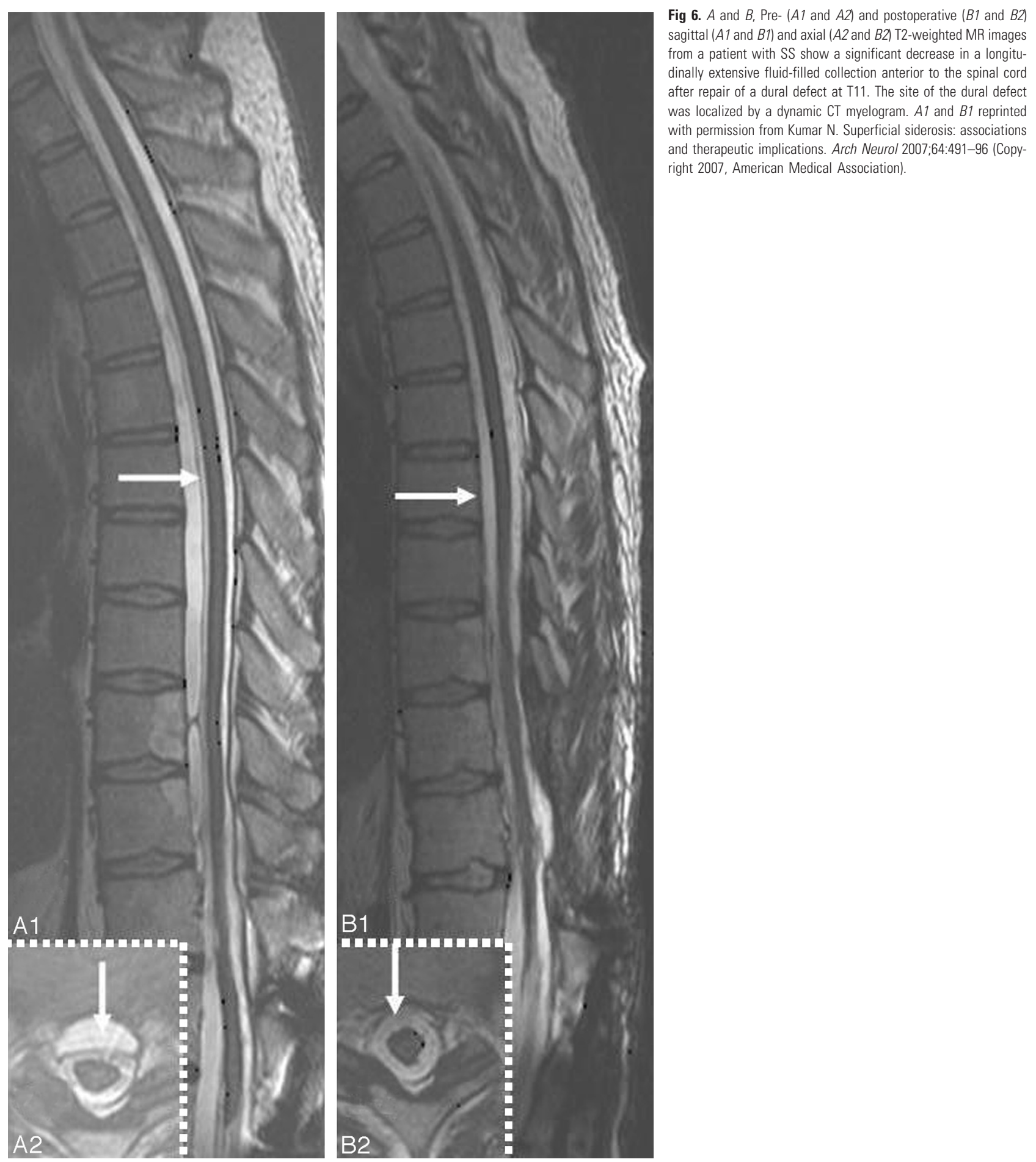

Fig 6. $A$ and $B$. Pre- $(A 1$ and $A 2)$ and postoperative $(B 1$ and $B 2$ sagittal ( $A 1$ and $B 1$ ) and axial ( $A 2$ and $B 2$ ) T2-weighted MR images with permission from Kumar N. Superficial siderosis: associations right 2007, American Medical Association).

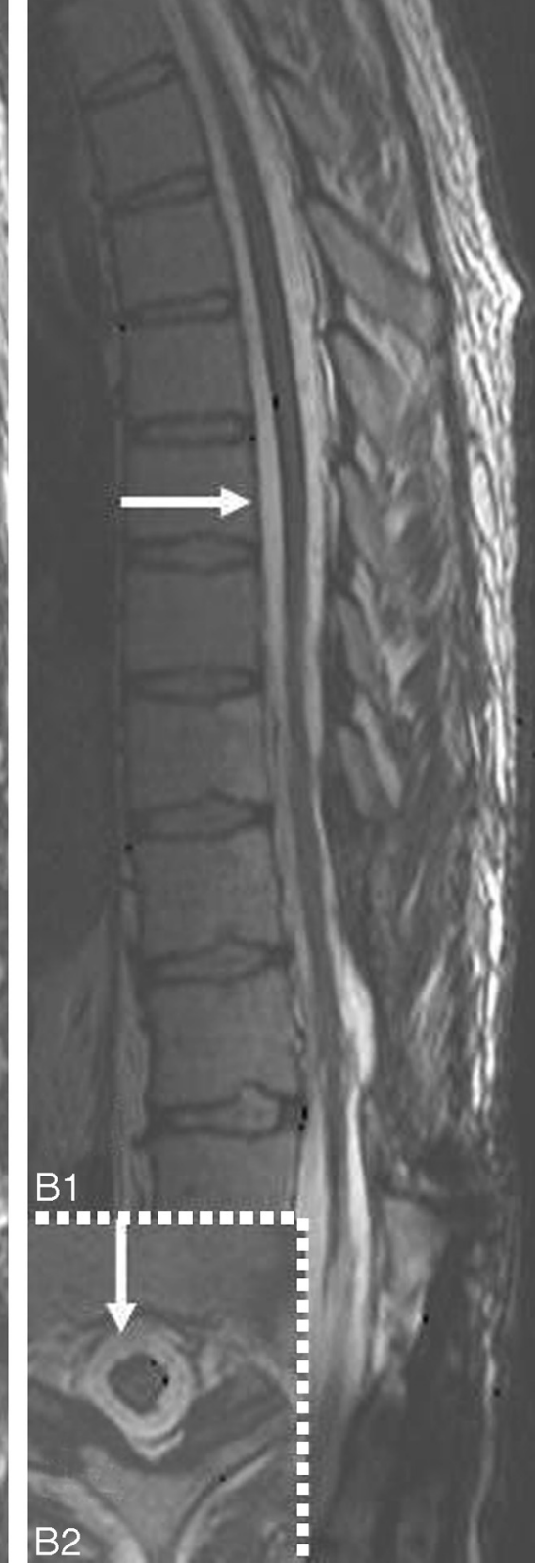

unruptured aneurysms. Progression despite resection of an arteriovenous fistula has also been reported. ${ }^{2}$ Vascular malformations that have been reported in association with SS include cerebral arteriovenous malformations (AVMs), spinal AVMs, spinal arteriovenous fistula, aneurysms, and cavernous malformations. ${ }^{9,16,25,33,57-60}$

Certain findings during neuroimaging in SS may mistakenly suggest the possibility of an underlying vascular malformation as a possible cause of chronic bleeding and prompt consideration of investigations like MR or conventional angiography. ${ }^{28}$ These include enhancement of the pial surface of

the cord on postgadolinium MR imaging suggestive of prominent vessels (Fig 11A1, -A2) or the presence of a prominent vessel or a CT myelogram (Fig $11 B$ ). The pathophysiology of the prominent vessels without vascular malformations seen during the work-up of SS is unknown. It has been suggested that the pial siderosis may contribute to sclerosis of the epidural plexus of veins, leading to venous hypertension. ${ }^{28}$ The more recently reported association between SS and CSF hypovolemia has raised the speculation that the increased CSF erythrocytes in SS could be because of intradural vascular engorgement that accompanies CSF hypotension. ${ }^{31,40}$ Imaging 

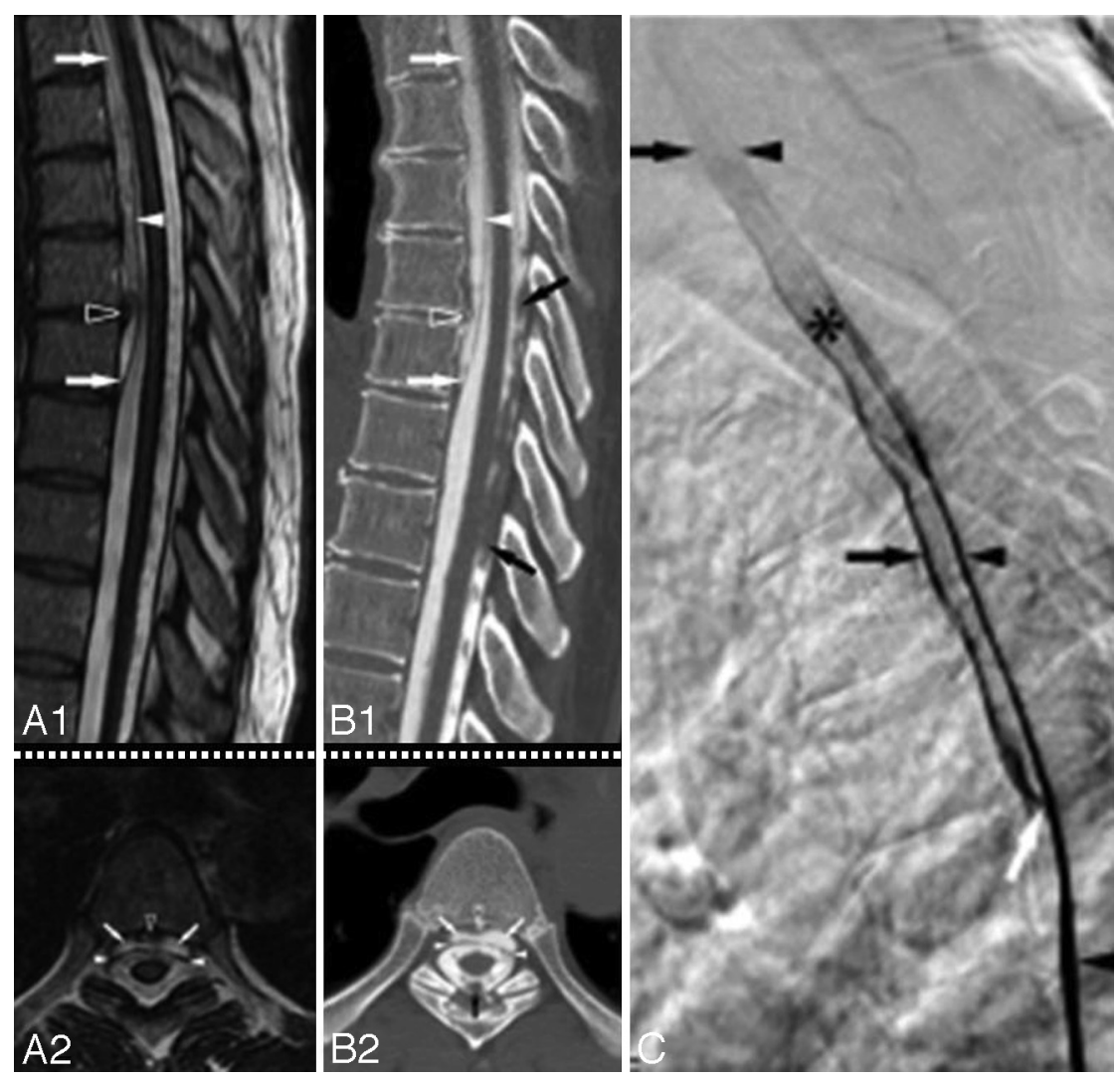

Fig 7. $A 1$ and $A 2$, Sagittal (A1) and axial (A2) T2-weighted MR images from a patient with SS show a cervicothoracic epidural fluid-filled collection (white arrows) and a T5-6 disk extrusion (black arrowhead) that displaces the dura (white arrowhead) posteriorly. $B 1$ and $B 2$, Corresponding sagittal (reformatted) (B1) and axial (B2) postmyelography CT images demonstrate opacification of the ventral epidural fluid by intrathecal contrast to the same degree as the CSF, thus confirming an active leak. Note partial calcification of the disk (black arrowhead) and the presence of a subarachnoid clot (black arrow). C, Lateral view of the thoracic spine acquired during digital substraction myelography with the patient in the prone position. Note cephalad extension of the contrast in the thecal sac (black arrowhead), focal extravasation of the contrast through a ventral dural tear at T5-6 (white arrow) into the epidural collection (black arrow), and onward cephalad extension in the epidural fluid collection. The asterisk indicates increased attenuation of the contrast because of the smaller volume of the epidural space compared with the subarachnoid space. In this patient, a ventral dural tear at T5-6 was surgically repaired, and a thoracic spine MR imaging performed 3 months later showed resolution of the ventral epidural fluid collection. ${ }^{36}$ Adapted with permission from J.M. Hoxworth. ${ }^{36}$
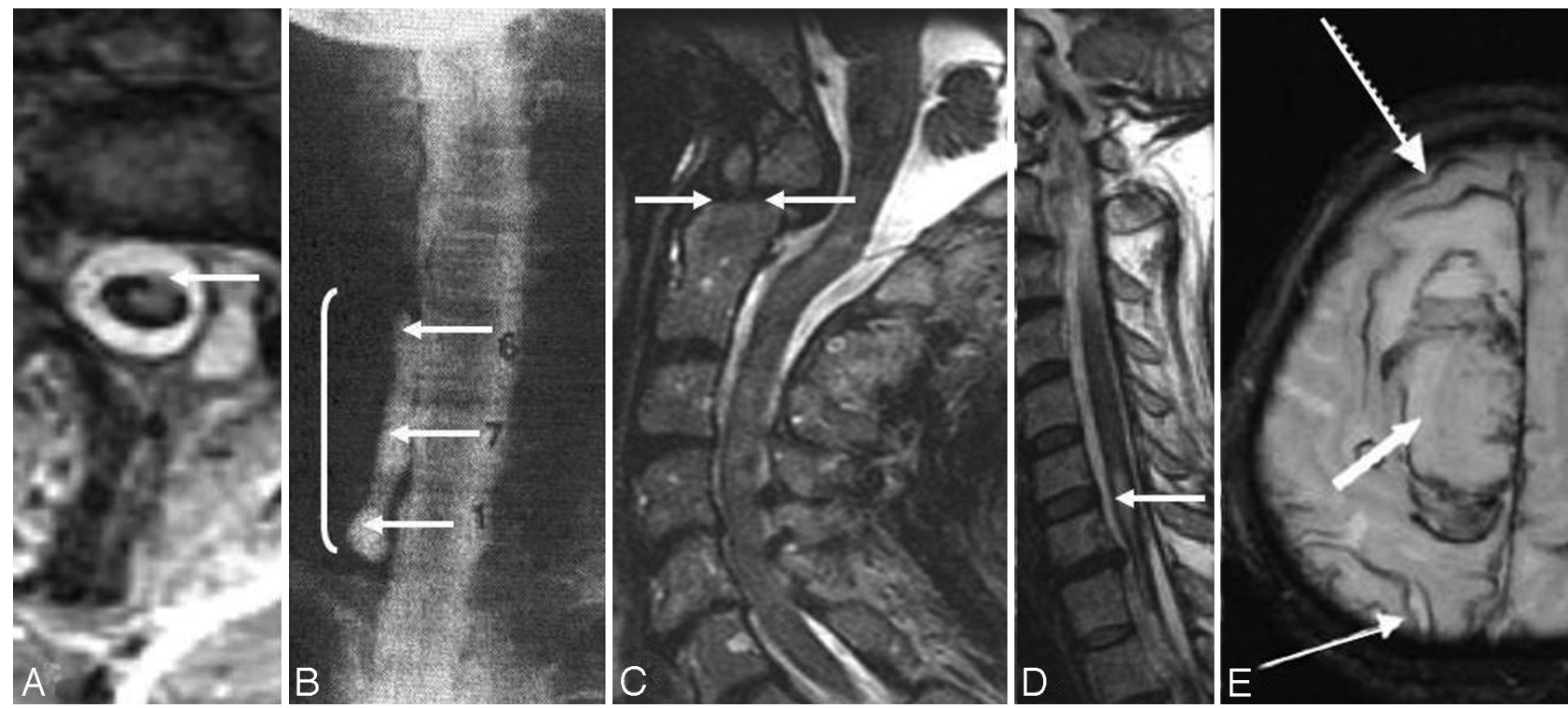

Fig 8. $A$, Axial T2-weighted MR image shows interruption of the rim of hypointensity around the spinal cord at the site of root avulsion. The interruption is likely due to the absence of the spinal cord pia mater at the site of root avulsion. $B, C 7$ to $T 1$ pseudomeningocele due to root avulsion seen on a cervical myelogram (same patient as the one shown in Fig $8 A$ ). $C$, T2-weighted sagittal cervical spine MR image from a patient with SS shows evidence of a prior odontoid fracture (same patient as the one shown in Fig 10C). $D$, Sagittal T2-weighted cervical spine MR image from a patient with SS shows an intramedullary T2 hypointensity due to myelomalacia secondary to prior trauma. $E$, T2* MR image from a patient with cerebral amyloid angiopathy shows a right frontal intracerebral hemorrhage (thick arrow) and hemosiderin deposition similar to that seen in superficial siderosis (thin arrows). C reprinted with permission from Kumar N, Cohen-Gadol AA, Wright RA, et al. Superficial siderosis. Neurology 2006;66:1144-52 (Copyright 2006, Wolters Kluwer Health). B and D reprinted with permission from Kumar N. Superficial siderosis: associations and therapeutic implications. Arch Neurol 2007;64:491-96 (Copyright 2007, American Medical Association). E adapted with permission from J. Linn. ${ }^{45}$

evidence of engorgement of intradural and epidural veins may be seen with CSF hypovolemia (Fig 9C2 and C3). ${ }^{40,61}$ Awareness of these associations in SS is important because it may obviate angiography.

\section{Other Potential Techniques}

Gadolinium-enhanced MR myelography and MR cisternography (MR imaging after intrathecal introduction of gadolinium) are additional techniques that have been used to improve 

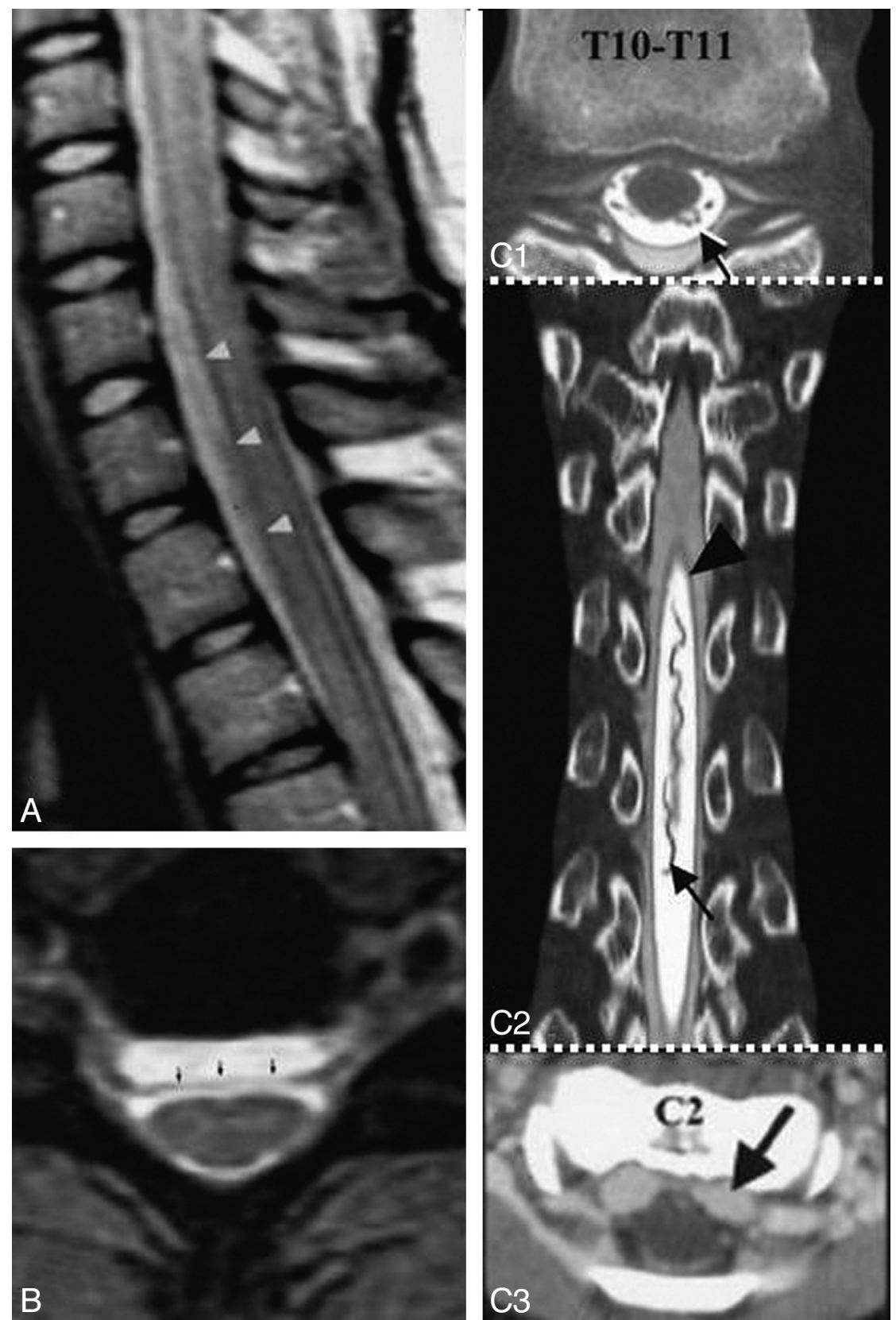

Fig 9. $A$, Sagittal T2-weighted cervicothoracic spine MR image from a patient with intracranial hypotension shows a ventral extradural collection from C6 to T2 (arrowhead), which is isointense with CSF on all imaging sequences. $B_{\text {, }}$ Axial T2-weighted gradient-echo MR image at C6-7 in a patient with intracranial hypotension shows a ventral extradural fluid collection separated from the thecal sac by a hypointense dura (arrows). $A$ and $B$ adapted with permission from B.M. Rabin. ${ }^{38} \mathrm{C} 1-C 3, \mathrm{~A}$ patient with craniospinal hypotension who had an epidural pseudomeningocele caused by a CSF leak at T8. C1, Axial CT myelogram of the thoracic spine shows the dura marginating an epidural pseudomeningocele (black arrow). C2, Coronal reformatted CT myelogram of the thoracic spine shows a tortuous dilated posterior thoracic spinal vein (black arrow), reminiscent of a dural arteriovenous fistula, and dura (arrowheads) separating intradural and epidural CSF. C3, Contrast-enhanced axial CT scan shows a dilated cervical epidural venous plexus. C1-C3 adapted with permission from J.L. Ulmer. ${ }^{61}$

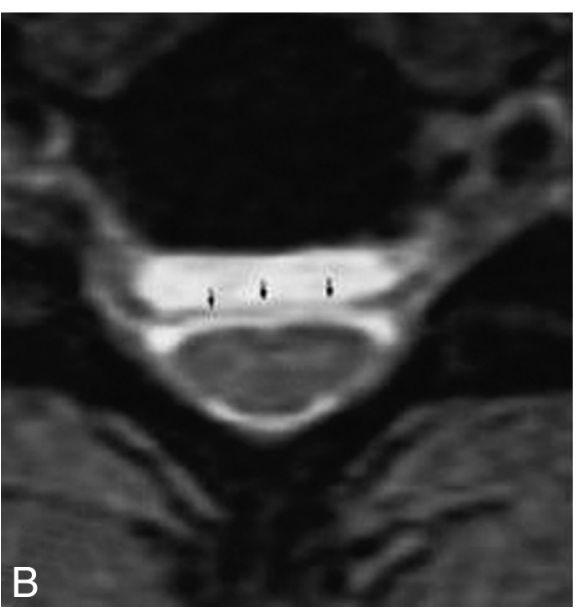

the detection of CSF leaks. ${ }^{62,63}$ Also reported is digital subtraction cisternography to identify intracranial CSF fistulas and digital substraction myelography to identify a dural tear in a patient with a cervical postoperative pseudomeningocele. ${ }^{64-66}$ In an isolated report, digital substraction myelography has been used to identify the site of a leak in a patient with SS and a cervicothoracic epidural fluid collection (Fig 7C). ${ }^{36}$

\section{Conclusions}

SS of the CNS is caused by chronic hemorrhage into the subarachnoid space. A clinical history of subarachnoid hemorrhage is often not present. This recurrent or persistent bleeding results in hemosiderin deposition in the subpial layers of the brain and spinal cord. The clinical presentation closely mimics a degenerative cerebellar disorder. A history of prior injury or intradural surgery is common. A potential source of bleeding should be carefully investigated by imaging the entire neuraxis. Despite extensive imaging, a source of bleeding is often not evident. MR imaging shows the characteristic marginal T2 hypointensity around the brain stem, cerebellum, and spinal cord and may also provide a clue to the possible etiology. Due to widespread use of MR imaging, SS is being increasingly recognized. A fluid-filled collection of variable dimensions is often seen on spine MR images or CT myelograms in patients with or without a prior history of injury or surgery. With longitudinally extensive collections, a CT myelogram may help localize the defect and direct the site of laminectomy. With large defects and high-flow leaks, a dynamic CT myelogram or digital substraction myelography may be needed to identify the dural defect. Dynamic CT myelography and digital substraction myelography are invasive techniques that are time-intensive and need a higher radiation dose. They should be used in carefully selected patients, and further research is needed to define their role in clinical practice. Surgical correc- 

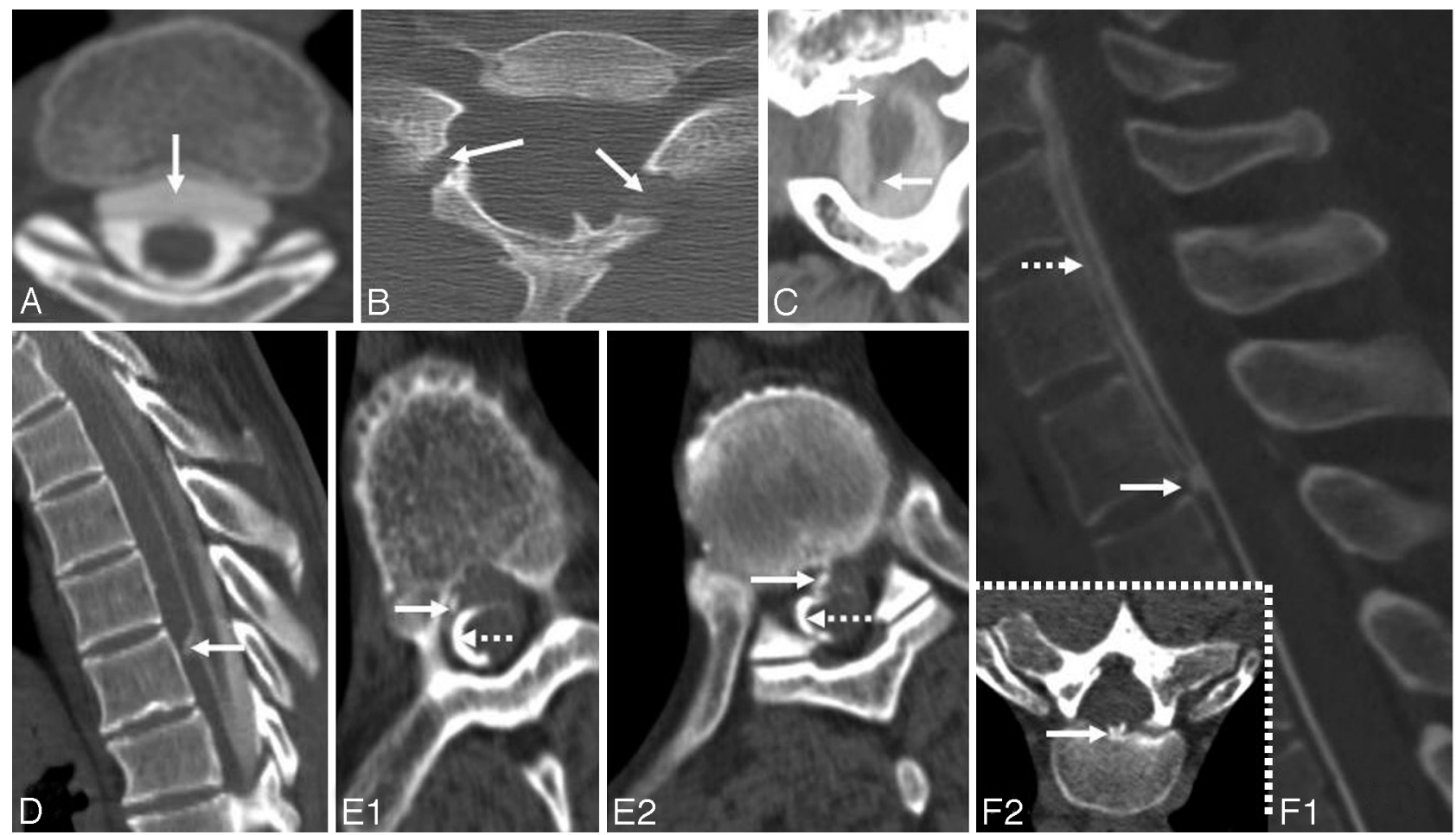

Fig 10. A, Fluid-filled intraspinal collection anterior to the cord on an axial cut of a thoracic spine CT myelogram. A transdural leak between C7 and T6 was present. A dynamic CT myelogram can localize the exact site of the defect and help direct the laminectomy site. B, Axial CT scan with bone windows from a patient with SS shows a bilaminar C7 fracture (same as patient shown in $4 C 1$ and C2). C. Axial cervical spine CT myelogram shows avulsed C2 nerve roots, which are directed in an anteroposterior direction and are seen as linear streaks with surrounding contrast (same patient as the one shown in Fig 8C). D. Dynamic CT myelogram from a patient with SS and a cervicothoracic epidural fluid-filled collection shows leakage of contrast through a dural tear at T1-2.29 E1, Dynamic CT myelogram shows leakage of contrast (arrow); the dotted arrow points to the intrathecal contrast. E2, Dynamic CT myelogram shows calcified disk

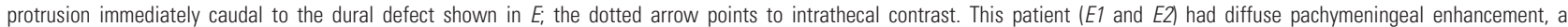
cervicothoracic epidural fluid collection, and CSF RBCs and xanthochromia, all of which resolved after repair of a dural defect identified at T7-8. F1, Reformatted sagittal cuts from a dynamic CT myelogram obtained in a patient with low-pressure headache without SS show a high-flow CSF leak (arrow) through a ventral midline defect located on the right side of a bilobed spiculated midline osteophyte at T2-3. F2, The osteophyte is shown on an axial thoracic spine CT. This patient also had a ventral epidural fluid-filled collection into which the contrast leaked through the dural defect (dotted arrow). C reprinted with permission from Kumar N. Superficial siderosis: associations and therapeutic implications. Arch Neurol 2007;64:491-96 (Copyright 2007, American Medical Association) and Kumar N, Lindell EP, Wilden JA, et al. Role of dynamic CT myelography in identifying the etiology of superficial siderosis. Neurology 2005;65:486-88 (Copyright 2005, Wolters Kluwer Health). E1 and E2 reprinted with permission from Kumar N, Lane Jl, Piepgras DG. Superficial siderosis: sealing the defect. Neurology 2009:72:671-73 (Copyright 2009, Wolters Kluwer Health).

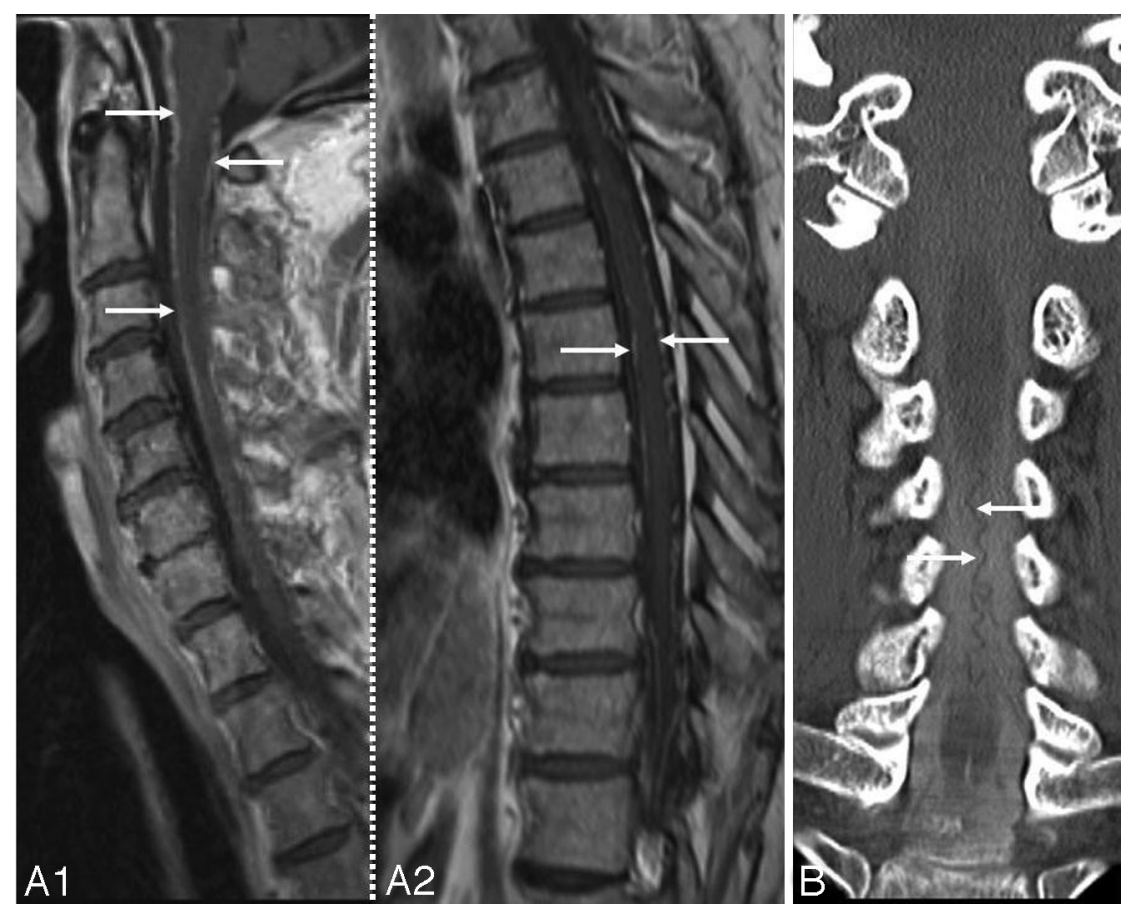

Fig 11. $A 1$ and $A 2$, Postcontrast sagittal T1-weighted cervical (A1) and thoracic (A2) MR images in a patient with SS show abnormal linear enhancement along the ventral and dorsal cord surface (same as the patient shown in $5 A$ and $B$ ). $B, C T$ myelogram in a patient with SS shows a posterior dilated pial venous plexus extending from the foramen magnum to C7. This prompted a cerebral and spinal angiography which showed no abnormality. A reprinted with permission from Kumar N, McKeon A, Rabinstein AA, et al. Superficial siderosis and CSF hypovolemia: the defect (dural) in the link. Neurology 2007;69:925-26 (Copyright 2007, Wolters Kluwer Health). 
tion of the cause of bleeding is a logical therapeutic strategy and holds promise for arresting disease progression. The relationship between SS and spontaneous craniospinal hypotension is of interest, but the precise mechanism of bleeding in patients with SS who have dural tears and intraspinal fluid collections is unknown.

\section{References}

1. Fearnley JM, Stevens JM, Rudge P. Superficial siderosis of the central nervous system. Brain 1995;118:1051-66

2. Kumar N, Cohen-Gadol AA, Wright RA, et al. Superficial siderosis. Neurology 2006;66:1144-52

3. Kumar N. Superficial siderosis: associations and therapeutic implications. Arch Neurol 2007;64:491-96

4. Koeppen AH, Michael SC, Li D, et al. The pathology of superficial siderosis of the central nervous system. Acta Neuropathol 2008;116:371-82. Epub 2008 Aug 12

5. Koeppen AH, Dentinger MP. Brain hemosiderin and superficial siderosis of the central nervous system. J Neuropathol Exp Neurol 1988;47:249-70

6. Koeppen AH, Hurwitz CG, Dearborn RE, et al. Experimental superficial siderosis of the central nervous system: biochemical correlates. J Neurol Sci 1992;112:38-45

7. Koeppen AH, Dickson AC, Chu RC, et al. The pathogenesis of superficial siderosis of the central nervous system. Ann Neurol 1993;34:646-53

8. Janss AJ, Galetta SL, Freese A, et al. Superficial siderosis of the central nervous system: magnetic resonance imaging and pathological correlation-case report. J Neurosurg 1993;79:756-60

9. Kumar A, Aggarwal S, Willinsky R, et al Posterior fossa surgery: an unusual cause of superficial siderosis. Neurosurgery 1993;32:455-57

10. Bonito V, Agostinis C, Ferraresi S, et al. Superficial siderosis of the central nervous system after brachial plexus injury: case report. J Neurosurg 1994;80:931-34

11. Tapscott SJ, Eskridge J, Kliot M. Surgical management of superficial siderosis following cervical nerve root avulsion. Ann Neurol 1996;40:936-40

12. Anderson NE, Sheffield S, Hope JK. Superficial siderosis of the central nervous system: a late complication of cerebellar tumors. Neurology 1999;52:163-69

13. McCarron MO, McKinstry CS, Gibson JM. Superficial siderosis 20 years after brain tumour. Lancet Neurol 2002;1:326

14. Konitsiotis S, Argyropoulou MI, Kosta P, et al. CNS siderosis after brachial plexus avulsion. Neurology 2002;58:505

15. McCarron MO, Flynn PA, Owens $\mathrm{C}$, et al. Superficial siderosis of the central nervous system many years after neurosurgical procedures. J Neurol Neurosurg Psychiatry 2003;74:1326-28

16. Leussink VI, Flachenecker P, Brechtelsbauer D, et al. Superficial siderosis of the central nervous system: pathogenetic heterogeneity and therapeutic approaches. Acta Neurol Scand 2003;107:54-61

17. Aquilina K, Kumar R, Lu J, et al. Superficial siderosis of the central nervous system following cervical nerve root avulsion: the importance of early diagnosis and surgery. Acta Neurochir (Wien) 2005;147:291-97

18. Cohen-Gadol AA, Atkinson PP, Krauss WE. Central nervous system superficial siderosis following spinal surgery. J Neurosurg Spine 2005;2:206-08

19. Gomori JM, Grossman RI, Bilaniuk LT, et al. High-field MR imaging of superficial siderosis of the central nervous system. J Comput Assist Tomogr 1985;9:972-75

20. Gomori JM, Grossman RI, Goldberg HI, et al. High-field spin-echo MR imaging of superficial and subependymal siderosis secondary to neonatal intraventricular hemorrhage. Neuroradiology 1987;29:339-42

21. Offenbacher H, Fazekas F, Reisecker F, et al. Superficial siderosis of the spinal cord: a rare cause of myelopathy diagnosed by MRI. Neurology 1991;41:1987-89

22. Bracchi M, Savoiardo M, Triulzi F, et al. Superficial siderosis of the CNS: MR diagnosis and clinical findings. AJNR Am J Neuroradiol 1993;14:227-36

23. Grunshaw ND, Blanshard KS, Hussain SS, et al. Superficial siderosis of the central nervous system: diagnosis by magnetic resonance imaging. Clin Radiol 1993;48:186-88

24. Pyhtinen J, Paakko E, Ilkko E. Superficial siderosis in the central nervous system. Neuroradiology 1995;37:127-28

25. Offenbacher H, Fazekas F, Schmidt R, et al. Superficial siderosis of the central nervous system: MRI findings and clinical significance. Neuroradiology 1996; 38( suppl 1):S51-56

26. Uchino A, Aibe $\mathrm{H}$, Itoh $\mathrm{H}$, et al. Superficial siderosis of the central nervous system: its MRI manifestations. Clin Imaging 1997;21:241-45

27. Cohen-Gadol AA, Krauss WE, Spinner RJ. Delayed central nervous system superficial siderosis following brachial plexus avulsion injury: report of three cases. Neurosurgl Focus 2004;16:E10

28. Wilden JA, Kumar N, Murali HR, et al. Unusual neuroimaging in superficial siderosis. Neurology 2005;65:489
29. Kumar N, Lindell EP, Wilden JA, et al. Role of dynamic CT myelography in identifying the etiology of superficial siderosis. Neurology 2005;65:486-88

30. Kumar N, McKeon A, Rabinstein AA, et al. Superficial siderosis and CSF hypovolemia: the defect (dural) in the link. Neurology 2007;69:925-26

31. Kumar N, Lane JI, Piepgras DG. Superficial siderosis: sealing the defect. Neurology 2009;72:671-73

32. Willeit J, Aichner F, Felber S, et al. Superficial siderosis of the central nervous system: report of three cases and review of the literature. J Neurol Sci 1992;111:20-25

33. Hughes JT, Oppenheimer DR. Superficial siderosis of the central nervous system: a report on nine cases with autopsy. Acta Neuropathol 1969;13:56-74

34. Sherwin I, Toll K. Superficial hemosiderosis of the central nervous system. Dis Nerv Syst 1972;33:413-17

35. Holle D, Sandalcioglu IE, Gizewski ER, et al. Association of superficial siderosis of the central nervous system and low pressure headache. J Neurol 2008;255:1081-82

36. Hoxworth JM, Patel AC, Bosch EP, et al. Localization of a rapid cerebrospinal fluid leak with digital substraction myelography. AJNR Am J Neuroradiol 2009;30:516-19

37. Mokri B, Piepgras DG, Miller GM. Syndrome of orthostatic headaches and diffuse pachymeningeal gadolinium enhancement. Mayo Clin Proc 1997;72:400-13

38. Rabin BM, Roychowdhury S, Meyer JR, et al. Spontaneous intracranial hypotension: spinal MR findings. AJNR Am J Neuroradiol 1998;19:1034-39

39. Mokri B. Spontaneous cerebrospinal fluid leaks: from intracranial hypotension to cerebrospinal fluid hypovolemia - evolution of a concept. Mayo Clin Proc 1999; 74:1113-23

40. Mokri B. Low cerebrospinal fluid pressure syndromes. Neurol Clin 2004;22:55-74

41. Revesz T, Earl CJ, Barnard RO. Superficial siderosis of the central nervous system presenting with longstanding deafness. $J \quad R$ Soc $\mathrm{Med}$ 1988;81:479-81

42. Koeppen AH, Barron KD. Superficial siderosis of the central nervous system: a histological, histochemical and chemical study. J Neuropathol Exp Neurol 1971;30:448-69

43. Kumar N, Bledsoe JM, Davis DH. Intracranial fluid-filled collection and superficial siderosis. J Neurol Neurosurg Psychiatry 2007;78:652-53

44. Kole MK, Steven D, Kirk A, et al. Superficial siderosis of the central nervous system from a bleeding pseudomeningocele: case illustration. J Neurosurg 2004;100:718

45. Linn J, Herms J, Dichgans M, et al. Subarachnoid hemosiderosis and superficial cortical hemosiderosis in cerebral amyloid angiopathy. AJNR Am J Neuroradiol 2008;29:184-86

46. Straube A, Dudel C, Wekerle G, et al. Polyradiculopathy in the course of superficial siderosis of the CNS. J Neurol 2001;248:63-64

47. Savoiardo M, Grisoli M, Pareyson D. Polyradiculopathy in the course of superficial siderosis of the CNS. J Neurol 2001;248:1099-100

48. Turner B, Wills AJ. Superficial siderosis associated with anterior horn cell dysfunction. J Neurol Neurosurg Psychiatry 2002;72:274-75

49. Messori A, Di Bella P, Herber N, et al. The importance of suspecting superficial siderosis of the central nervous system in clinical practice. J Neurol Neurosurg Psychiatry 2004;75:188-90

50. Fishman RA, Dillon WP. Dural enhancement and cerebral displacement secondary to intracranial hypotension. Neurology 1993;43:609-11

51. Good DC, Ghobrial M. Pathologic changes associated with intracranial hypotension and meningeal enhancement on MRI. Neurology 1993;43:2698-700

52. Mokri B, Parisi JE, Scheithauer BW, et al. Meningeal biopsy in intracranial hypotension: meningeal enhancement on MRI. Neurology 1995;45: 1801-07

53. Kwartler JA, De La Cruz A, Lo WW. Superficial siderosis of the central nervous system. Ann Otol Rhinol Laryngol 1991;100:249-50

54. Parnes SM, Weaver SA. Superficial siderosis of the central nervous system: a neglected cause of sensorineural hearing loss. Otolaryngol Head Neck Surg 1992;107:69-77

55. Pinkston JW, Ballinger WE Jr, Lotz PR, et al. Superficial siderosis: a cause of leptomeningeal enhancement on computed tomography. J Comput Assist Tomogr 1983;7:1073-76

56. Luetmer PH, Mokri B. Dynamic CT myelography: a technique for localizing high-flow spinal cerebrospinal fluid leaks. AJNR Am J Neuroradiol 2003;24:1711-14

57. Lai MT, Ohmichi T, Yuen K, et al. Superficial siderosis of the central nervous system: a case with an unruptured intracranial aneurysm. J Laryngol Otol 1995;109:549-52

58. Schievink WI, Apostolides PJ, Spetzler RF. Surgical treatment of superficial siderosis associated with a spinal arteriovenous malformation: case report. J Neurosurg 1998;89:1029-31

59. Hsu WC, Loevner LA, Forman MS, et al. Superficial siderosis of the CNS asso- 
ciated with multiple cavernous malformations. AJNR Am J Neuroradiol 1999;20:1245-48

60. Haroun RI, Li KW, Rigamonti D. Surgical resection of a cerebral arteriovenous malformation for treatment of superficial siderosis: case report. Surg Neurol 2000;53:554-58

61. Burtis MT, Ulmer JL, Miller GA, et al. Intradural spinal vein enlargement in craniospinal hypotension. AJNR Am J Neuroradiol 2005;26:34-38

62. Tali ET, Ercan N, Krumina G, et al. Intrathecal gadolinium (gadopentetate dimeglumine) enhanced magnetic resonance myelography and cisternography: results of a multicenter study. Invest Radiol 2002;37:152-59

63. Jinkins JR, Rudwan M, Krumina G, et al. Intrathecal gadolinium-enhanced MR cisternography in the evaluation of clinically suspected cerebrospinal fluid rhinorrhea in humans: early experience. Radiology 2002;222:555-59

64. Byrne JV, Ingram CE, MacVicar D, et al. Digital subtraction cisternography: a new approach to fistula localisation in cerebrospinal fluid rhinorrhoea. $\mathrm{JNeu}$ rol Neurosurg Psychiatry 1990;53:1072-75

65. Wakhloo AK, van Velthoven V, Schumacher M, et al. Evaluation of MR imaging, digital subtraction cisternography, and CT cisternography in diagnosing CSF fistula. Acta Neurochir (Wien) 1991;111:119-27

66. Phillips CD, Kaptain GJ, Razack N. Depiction of a postoperative pseudomeningocele with digital subtraction myelography. AJNR Am J Neuroradiol 2002;23:337-38 